\title{
Handling Uncertainties of Robot Manipulators and Active Vision by Constraint Propagation
}

\author{
Christopher C. Yang* \\ Department of Systems Engineering \\ and Engineering Management \\ The Chinese University of Hong Kong, Hong Kong \\ Frank W. Ciarallo \\ Department of Systems and Industrial Engineering \\ The University of Arizona, USA \\ Michael M. Marefat \\ Department of Electrical and Computer Engineering \\ The University of Arizona, USA \\ Received 1 June 2001; revised 30 May 2002; accepted 13 June 2002
}

\begin{abstract}
Joint errors are inevitable in robot manipulation. These uncertainties propagate to give rise to translational and orientational errors in the position and orientation of the robot end-effector. The displacement of the active vision head mounted on the robot endeffector produces distortion of the projected object on the image. Upon active visual inspection, the observed dimension of a mechanical part is given dimension by the measurement on the projected edge segment on the image. The difference between the observed dimension and the actual dimension is the displacement error in active vision. For different motion of the active vision head, the resulting displacement errors are different. Given the uncertainties of the robot manipulator's joint errors, constraint propagation can be employed to assign the motion of the active sensor in order to satisfy the tolerance of the displacement errors for inspection. In this article, we define the constraint consistency and network satisfaction in the constraint network for the problem of displacement errors in active vision. A constraint network is a network where the nodes represent variables, or constraints, and the arcs represent the relationships between the output variables and the input variables of the constraints. In the displacement errors problem, the tolerance of the displacement errors and the translational and orientational errors of robot manipulators have interval values while the sensor motion has real values. Constraint propagation is developed to propagate the tolerance of displacement errors in the hierarchical interval constraint network in order to find the feasible robot motion. ๑ 2002 Wiley Periodicals, Inc.
\end{abstract}

*To whom all correspondence should be addressed; e-mail: yang@se.cuhk.edu.hk.

Journal of Robotic Systems 19(9), 427-441 (2002) (c) 2002 Wiley Periodicals, Inc.

Published online in Wiley InterScience (mww.interscience.wiley.com). • DOI: 10.002/rob.10051 


$\begin{array}{ll}\text { List of Symbols } & \\ \mathbf{X} & \text { variable } \\ \mathbf{X} & \text { interval assigned for } \mathbf{X} \\ x_{\mathrm{up}} & \text { upper limit of } \mathrm{X} \\ x_{\text {low }} & \text { lower limit of } \mathrm{X} \\ x & \text { any value in } \mathrm{X} \\ t x, t y, t z & \text { translation in } x, y, z \text { directions } \\ \psi, \theta, \phi & \text { angle of rotation about } x, y, z \text { axes } \\ \Delta t x, \Delta t y, \Delta t z & \text { translation errors of end effectors } \\ \Delta \psi, \Delta \theta, \Delta \phi & \text { orientational errors of end effectors } \\ x_{w}, y_{w}, z_{w} & \text { coordinates of object in the world } \\ u, v & \text { coordinate system } \\ & \text { projected coordinates on the } \\ \varepsilon_{d u_{i}, \varepsilon_{d v_{i}}} & \text { image plane } \\ C_{i} & \text { horizontal and vertical displacement } \\ f() & \text { errors of point } i \text { in active vision } \\ F() & \text { constraints } \\ & \text { constraint function } \\ & \text { interval constraint function }\end{array}$

\section{INTRODUCTION}

Joint errors are inevitable in robot manipulation; these errors propagate to the displacement errors in active vision. In active visual inspection, the displacement errors are critical in the accuracy of dimensioning. Assignment of the active sensor motion without careful investigation will exaggerate the errors in dimensioning and result in rejecting parts unnecessarily. Several researchers have investigated the uncertainties in robot manipulation. Su et al. ${ }^{11}$ presented a methodology for manipulating and propagating spatial uncertainties in a robotic assembly system for generating executable actions for accomplishing a desired task. Renders et al. ${ }^{9}$ presented a calibration technique of robots based on a maximum likelihood approach for the identification of errors of position and orientation. Chen et al. ${ }^{1}$ identified and parameterized the sources that contribute to the positioning error and estimated the values of the parameters. Smith et al. ${ }^{10}$ presented a method for explicitly representing and manipulating the uncertainty associated with the transformation between coordinate frames representing the relative locations of objects. Sensors are used to reduce this uncertainty. However, little work has been done on handling uncertainties of robot manipulators for active visual inspection.

In robotics, the relationships between translation and orientation parameters, dimensions, and errors can be modelled by a constraint network. These relationships are described by constraint functions and the variables in these constraint functions may have interval or real values. Some variables have interval values. For example, the tolerance of displacement errors in active vision have interval values and the errors in translation or orientation of a robot arm have values of $\pm \Delta t x, \pm \Delta t y, \pm \Delta t z, \pm \Delta \psi, \pm \Delta \theta$, and $\pm \Delta \phi$. Some other variables have real values. For example, the translations and orientations assigned to the movement of a robot arm have real values. Given all the constraints, it is desirable to find feasible interval and real values for all the involved variables such that all the constraints are satisfied.

\subsection{Related Work in Constraints}

Constraint satisfaction problems (CSPs) are often formulated as AI tasks in which values are assigned to variables subject to a set of constraints. Constraint specifications represent the relationships among the variables. A constraint network is a declarative structure that consists of nodes and arcs where nodes represent the variables or the constraints and arcs represent relationships between the variables and the constraints. The variables are labeled by intervals, or sets of possible values. The constraints include any type of mathematical operation or binary relationships. The mathematical operations can be multiple inputs/single output (MISO) or single input/single output (SISO). Constraint propagation is utilized to derive inferences about the variable quantities in the network. For different types of variables and for different definitions of satisfaction in constraint satisfaction problems, different propagation techniques can be formulated. For applications in tolerance design and robotics, the variables are labeled by intervals or by real values and the constraints are n-ary mathematical operations.

In a constraint network, there is a set of constraints, each of which has an output variable and a set of input variables. The function that relates the variables in a constraint is known as a constraint function, and it can be expressed as: $\mathbf{X}_{\mathbf{k}}=f\left(\mathbf{X}_{\mathbf{1}}, \mathbf{X}_{\mathbf{2}}, \ldots, \mathbf{X}_{\mathbf{n}}\right)$ where $\mathbf{X}_{\mathbf{k}}$ is the output variable and $\mathbf{X}_{\mathbf{i}}$ is the $i$ th input variable. $n$ is the number of entities that are related by the mathematical operation (constraint function) to $\mathbf{X}_{\mathbf{k}}$. Each input or output variable can be the input variable or the output variable for other constraints.

Dechter and Pearl ${ }^{4,5}$ developed a method of generating heuristic guidance for the order of value assignments, based on the sparseness in the constraint network and the simplicity of tree-structured CSPs. A backtracking search algorithm is utilized to search for one or all solutions that assign to each variable a value which satisfies all the constraints. Mackworth 
and Freduer ${ }^{8}$ analyzed the time complexity of several node, arc, and path consistency algorithms in CSPs. However, the domains of the variables considered by Dechter and Pearl, and Mackworth and Freduer, are discrete, finite sets. Real intervals are not considered.

Ladkin and Reinefeld ${ }^{7}$ developed a technique for solving qualitative interval constraint problems, but their constraints are binary relations on intervals rather than $\mathrm{n}$-ary mathematical operations on intervals.

Davis and Hyvonen have investigated the interval constraint satisfaction problems (ICSPs), where constraints are n-ary mathematical operations and the intervals are real intervals. Davis adapted the Waltz filtering algorithm ${ }^{3}$ for screening impossible values from the variable domain to solve the ICSPs. However, the Waltz filtering algorithm cannot determine global solutions in general. Hyvonen ${ }^{6}$ used the tolerance propagation approach, which combines consistency techniques based on the topology of the constraint net with techniques of interval arithmetic, to solve the ICSPs. This method makes it possible to determine global solutions for ICSPs; however, its definition of consistency and satisfaction of the constraint network are not appropriate to some classes of problems in mechanical design and robotics. Furthermore, the variables in $\left(\right.$ Davis $^{3}$, and Hyvonen ${ }^{6}$ ) are restricted to interval values only. Differences between the definitions and the applications will be discussed in detail in the later sections. In this work, a new approach is presented for construction of constraint networks and propagation of constraints to accommodate interval and real valued variables. We investigate application of the proposed techniques to solve problems in robotics and active visual inspection.

\subsection{Handling Robot Manipulator Uncertainties by Hierarchical Constraint Networks}

By using hierarchical constraint networks, we have developed techniques to propagate interval values from level to level in a network in either forward or backward directions. ${ }^{13}$ For example, in tolerance design the dimensions of the entities and their tolerances and the parameters of the mechanical design are at the lowest level of the network, and the functional requirements are at the highest level. Propagation from the lowest level to the highest level uses forward propagation and from the highest level to the lowest level uses backward propagation. By using the hierarchical constraint networks, we can model the relationship between the displacement errors and the motion of the active vision head. Based on the developed constraint network, we employ the constraint propagation to satisfy all the constraints such that the tolerance of displacement errors of an active vision sensor is satisfied.

The research in the area of ICSPs has lacked a definition of consistency appropriate for application to control displacement errors in active visual inspection. In addition, the variables have been restricted to intervals. In this work, consistency has been redefined. The contributions of this work can be summarized as follows:

- Develop systematic methods for developing and handling constraint satisfaction based on hierarchical constraint networks with interval and real variables.

- Present new constraint consistency and network satisfaction definitions.

- Develop propagation techniques based on the new definitions for a constraint network with interval and real variables.

- Apply the developed techniques on controlling dimensioning errors due to sensor displacement in active vision.

\section{UNCERTAINTIES IN ROBOT MANIPULATORS AND ACTIVE VISUAL INSPECTION}

The uncertainties in robot manipulation are the result of joint errors which cannot be avoided and will propagate to give rise to translational and orientational errors in the position and orientation of the robot endeffectors. These propagated errors may cause the failure of an assigned assembly task or inaccurate data collection from the attached sensor. Many researchers have investigated the characteristics of the uncertainties in robot manipulators and sought techniques to control them in order to minimize these errors. In this article, constraint networks are utilized to adjust the path in order to make sure the allowed tolerances of the end-effector position are satisfied. The joint motion of the end effector is derived based on the transformation as follows:

$$
T=\operatorname{Trans}(t x, t y, t z) \operatorname{Rot}(x, \psi) \operatorname{Rot}(y, \theta) \operatorname{Rot}(z, \phi)
$$

Given the translational and orientational errors of the joint motion, $\Delta t x, \Delta t y, \Delta t z, \Delta \psi, \Delta \theta$, and $\Delta \phi$, the transformation is derived as Eq. (1).

$$
\begin{aligned}
T^{\prime}= & \operatorname{Trans}(t x+\Delta t x, t y+\Delta t y, t z+\Delta t z) \\
& \times \operatorname{Rot}(x, \psi+\Delta \psi) \operatorname{Rot}(y, \theta+\Delta \theta) \operatorname{Rot}(z, \phi+\Delta \phi)
\end{aligned}
$$


A cylindrical coordinate arm and a robot arm with six degrees of freedom are used for illustration here. The cylindrical coordinate arm has one rotary motion and two linear motions. The robot arm with six degrees of freedom has three rotary motions and three linear motions.

\subsection{Cylindrical Coordinate Arm}

A cylindrical coordinate arm is shown in Figure 1. The transformations relating the end effector to joint motions for a cylindrical coordinate arm is formulated as follows:

$T=\operatorname{Trans}(0,0, t z) \operatorname{Rot}(z, \alpha) \operatorname{Trans}(\operatorname{tr} \cos \beta, \operatorname{tr} \sin \beta, 0)$

$T=\left[\begin{array}{cccc}1 & 0 & 0 & 0 \\ 0 & 1 & 0 & 0 \\ 0 & 0 & 1 & t z \\ 0 & 0 & 0 & 1\end{array}\right]\left[\begin{array}{cccc}\cos \alpha & -\sin \alpha & 0 & 0 \\ \sin \alpha & \cos \alpha & 0 & 0 \\ 0 & 0 & 1 & 0 \\ 0 & 0 & 0 & 1\end{array}\right]$

$\times\left[\begin{array}{cccc}1 & 0 & 0 & \operatorname{tr} \cos \beta \\ 0 & 1 & 0 & \operatorname{tr} \sin \beta \\ 0 & 0 & 1 & 0 \\ 0 & 0 & 0 & 1\end{array}\right]$
$=\left[\begin{array}{cccc}\cos \alpha & -\sin \alpha & 0 & \operatorname{tr} \cos (\alpha+\beta) \\ \sin \alpha & \cos \alpha & 0 & \operatorname{tr} \cos (\alpha+\beta) \\ 0 & 0 & 1 & t z \\ 0 & 0 & 0 & 1\end{array}\right]$

$\left[x^{\prime}, y^{\prime}, z^{\prime}, 1\right]^{T}=T[x, y, z, 1]^{T}$
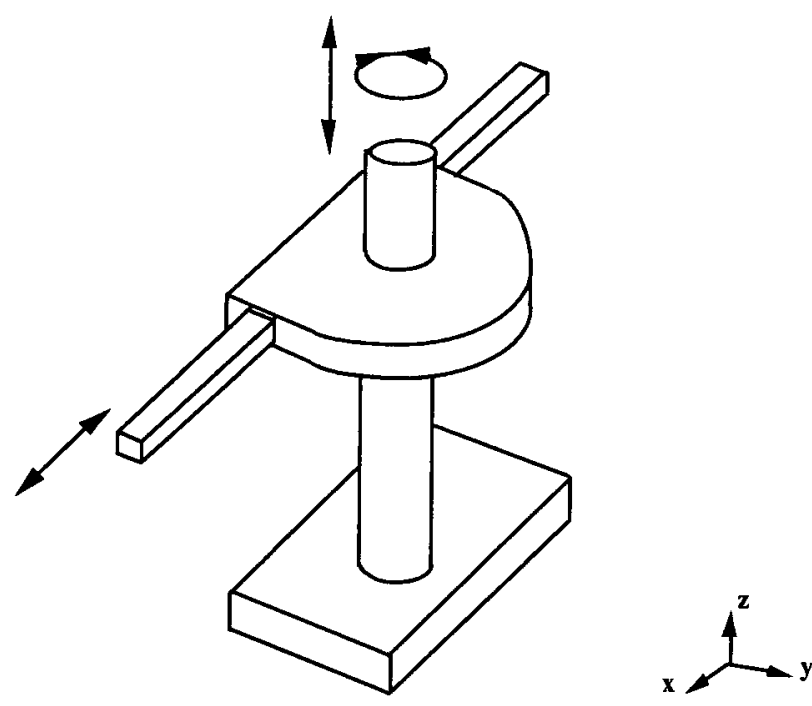

Figure 1. A cylindrical coordinate arm. where $[x, y, z]$ is the initial or home position of the end effector

$\left[x^{\prime}, y^{\prime}, z^{\prime}\right]$ is the transformed or resulting position

$t z$ and $t r$ are the translation parameters in $z$ direction and the direction with an angle of $\beta$ with the $x$ axis on the $x-y$ plane, respectively

$\beta$ is the initial angle between the robot arm and the $x$ axis on the $x-y$ plane

$\alpha$ is the rotation parameter of the robot arm about the $z$ axis

The uncertainties in the movement of the cylindrical coordinate arm are due to the joint motion errors, $\Delta t z$ and $\Delta t r$, and the orientational error, $\Delta \alpha$. The transformation matrix accounting for the translational and orientation errors, $T^{\prime}$, is formulated as follows:

$$
\begin{aligned}
& T^{\prime}= \\
& {\left[\begin{array}{cccc}
\cos (\alpha+\Delta \alpha) & -\sin (\alpha+\Delta \alpha) & 0 & t r^{\prime} \cos (\alpha+\Delta \alpha+\beta) \\
\sin (\alpha+\Delta \alpha) & \cos (\alpha+\Delta \alpha) & 0 & t r^{\prime} \sin (\alpha+\Delta \alpha+\beta) \\
0 & 0 & 1 & t z^{\prime} \\
0 & 0 & 0 & 1
\end{array}\right]}
\end{aligned}
$$

where $\Delta \alpha$ is the joint error in the rotation about the $z$ axis

$\Delta t r$ is the joint error in the translation along the direction with an angle of $\beta$ with the axis on the $x-y$ plane

$\Delta t z$ is the joint error in the translation along the $z$ direction

\subsection{Displacement Errors in Active Vision}

Displacement errors come about because of the displacement of an active vision head from its expected position and orientation. They represent the difference between the imaged position of a geometric entity such as a point and the expected position of the geometric entity on the image if there is no displacement of the active vision head (Fig. 2). Mechanical displacements of active vision heads are not avoidable. Thus, in a vision task involving multiple sensing steps, we need to either have the ability to calibrate the vision sensor in each step, or we need to have methods that can accommodate possible displacement errors. An example of using active vision sensing for dimensional inspection of shape feature attributes 


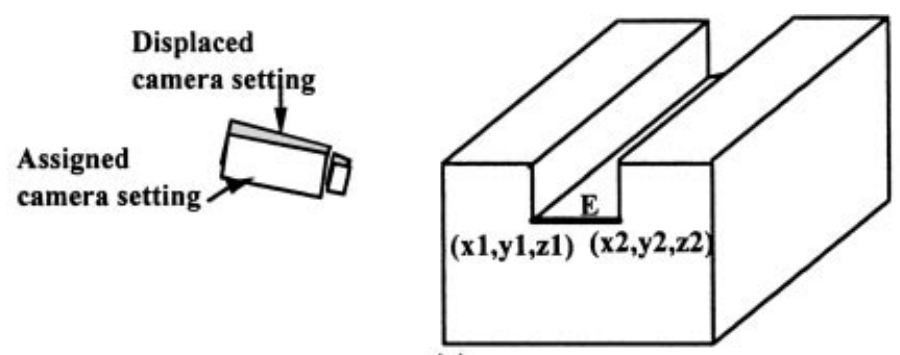

(a)

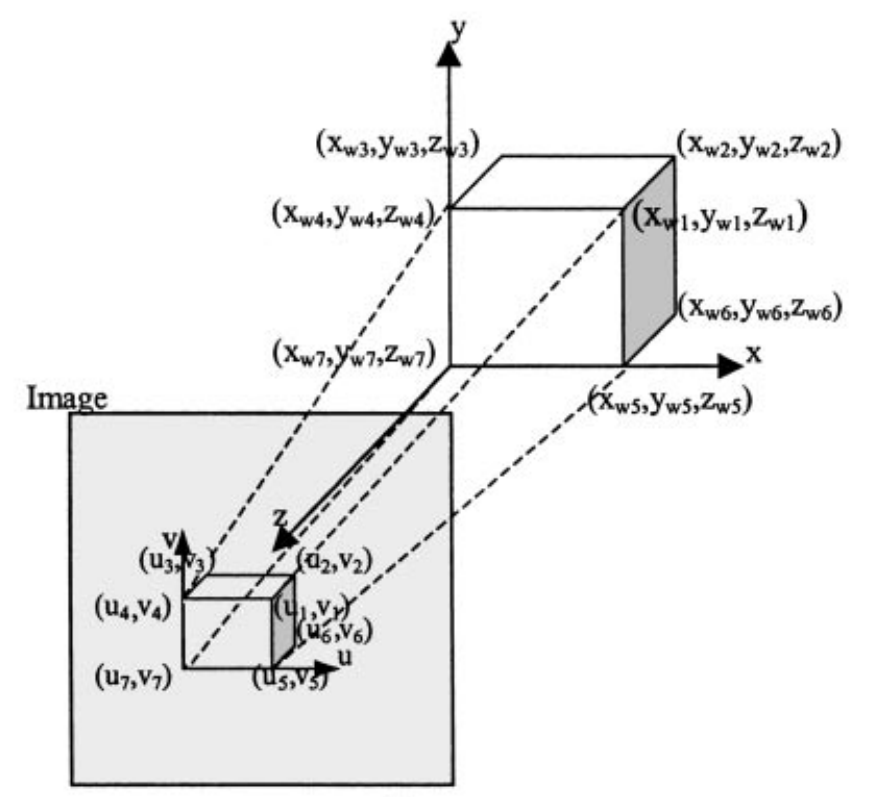

(b)

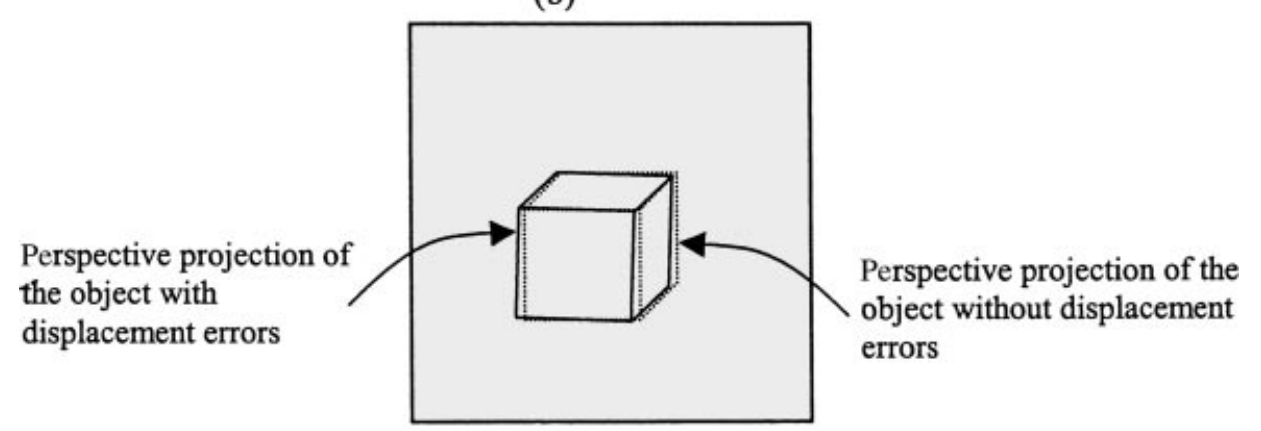

(c)

Figure 2. (a) An edge segment, $E$, which is the width of the slot on the component, with end points, $(x 1, y 1, z 1)$ and $(x 2, y 2, z 2)$, is dimensioned by the camera with the assigned setting as shown. However, due to displacement error, the measurement could be undesirably affected by the displaced camera setting. (b) Perspective projection: An object point $\left(x_{w i}, y_{w i}, z_{w i}\right)$ is projected onto the image plane as $\left(u_{i}, v_{i}\right)$ where $i=1,2,3, \ldots, 7$. (c) Object projected onto the image plane using perspective project with (solid lines) and without (dotted lines) displacement errors.

of machined components is discussed by Yang et al. ${ }^{2,12-15}$ Having a good understanding of displacement errors and a good methodology to account for them is important for the accuracy of the automated inspection of an entity's dimensions. In this article, we utilize constraint networks to determine a sensor setting at which displacement errors are within the desired tolerance level in inspection. 


\subsubsection{Translational and Orientational Errors in Perspective Images}

In active visual inspection, different sensor settings are used to position the active head to obtain and inspect various dimensions of interest. The desired positions and orientations of the head are provided to the servo control that accomplishes the sensor placement task by a sequence of movements. However, errors in the final position and orientation are common. The image of the inspected part obtained from the sensor, the entities observable from that image and the measured dimensions of the entities all depend on the sensor location and viewing direction. If the sensor location and orientation are different from the planned sensor setting (i.e., there is sensor displacement), the same entities may be observable, but because of the displacement, the dimensions derived from the image will be inaccurate. The difference between the observed dimensions and the actual dimensions is defined as displacement error. The horizontal and vertical displacements of the projected points on the two-dimensional image are in terms of the six orientation and translation parameters of the sensor setting, the focal length of the sensor, the translational and orientational errors, and the threedimensional coordinates of the model. The analysis in this section yields a better understanding of dimensional measurement incorporating error due to displacement of the active sensor. These results are useful in reducing the occurrence and impact of such errors.

Given the orientation and location of the sensor and the world coordinates of the object, we can compute the two-dimensional coordinates of the projected object on the image plane as follows:

$$
\begin{aligned}
& {\left[\begin{array}{c}
x_{I} \\
y_{I} \\
z_{I} \\
\chi
\end{array}\right]=P_{\text {per }} Q\left[\begin{array}{c}
x_{w} \\
y_{w} \\
z_{w} \\
1
\end{array}\right]} \\
& u=x_{I} / \chi, \text { and } v=y_{I} / \chi
\end{aligned}
$$

where $\left[x_{w}, y_{w}, z_{w}\right]^{T}$ are the coordinates of the object in the world coordinate system, $(u, v)$ are the projected coordinates in the image plane, $P_{\text {per }}$ is the matrix for perspective projection, and $Q$ is the transformation matrix between the world coordinates and the image coordinate system.

$$
P_{\text {per }}=\left[\begin{array}{cccc}
1 & 0 & 0 & 0 \\
0 & 1 & 0 & 0 \\
0 & 0 & 1 & -\frac{1}{f} \\
0 & 0 & 0 & 1
\end{array}\right] ; \quad Q=\left[\begin{array}{cccc}
r_{11} & r_{12} & r_{13} & t_{x} \\
r_{21} & r_{22} & r_{23} & t_{y} \\
r_{31} & r_{32} & r_{33} & t_{z} \\
0 & 0 & 0 & 0
\end{array}\right]
$$

$f$ is the focal length of the sensor, $\left[r_{i j}\right]_{3 \times 3}$ is the rotation submatrix in terms of the orientation parameters, and $\left[t_{k}\right]_{3 \times 1}$ is the translation submatrix in terms of the translation parameters.

If the sensor is displaced, the correct coordinates of the projected points must be computed with modification of the $Q$ matrix because the rotation and translation parameters are distorted due to displacement. Thus, a matrix $Q^{\prime}$ must be substituted for $Q$ in Equation (5) to compensate. $Q^{\prime}$ is in terms of the three translational errors, $d x, d y, d z$, and three orientational errors, $\delta x, \delta y$, and $\delta z$, and the original translational and orientational parameters. The perspective matrix is unchanged because its only parameter, $f$, is fixed.

$$
\begin{aligned}
Q^{\prime}= & Q+\Delta Q \\
= & \operatorname{Trans}(d x, d y, d z) \operatorname{Rot}(x, \delta x) \\
& \times \operatorname{Rot}(y, \delta y) \operatorname{Rot}(z, \delta z) Q
\end{aligned}
$$

where Trans $(d x, d y, d z)$ is a transformation representing a translation by $d x, d y$, and $d z$. $\operatorname{Rot}(x, \delta x)$, $\operatorname{Rot}(y, \delta y)$, and $\operatorname{Rot}(z, \delta z)$ are transformations representing a differential rotation about the $x, y$, and $z$ directions, respectively. $\Delta$ is given as:

$$
\Delta=\left[\begin{array}{cccc}
0 & -\delta z & \delta y & d x \\
\delta z & 0 & -\delta x & d y \\
-\delta y & \delta x & 0 & d z \\
0 & 0 & 0 & 0
\end{array}\right]
$$

Therefore:

$$
Q^{\prime}=Q+\Delta Q=\left[\begin{array}{cccc}
r_{11}-r_{21} \delta z+r_{31} \delta y & r_{12}-r_{22} \delta z+r_{32} \delta y & r_{13}-r_{23} \delta z+r_{33} \delta y & t_{x}-\delta z t_{y}+\delta y t_{z}+d x \\
r_{21}+r_{11} \delta z-r_{31} \delta x & r_{22}+r_{12} \delta z-r_{32} \delta x & r_{23}+r_{13} \delta z-r_{33} \delta x & t_{y}+\delta z t_{x}-\delta x t_{z}+d y \\
r_{31}-r_{11} \delta y+r_{21} \delta x & r_{32}-r_{12} \delta y+r_{22} \delta x & r_{33}-r_{13} \delta y+r_{23} \delta x & t_{z}-\delta y t_{x}+\delta x t_{y}+d z \\
0 & 0 & 0 & 1
\end{array}\right]
$$


As a result, the displaced image coordinates $\left(u^{\prime}, v^{\prime}\right)$ are computed by using Eqs. (5), (6), and (7) and are given as follows:

$$
u^{\prime}=\frac{f(S 1+S 3 \delta y-S 2 \delta z+d x)}{f-(S 3+S 2 \delta x-S 1 \delta y+d z)}
$$

and

$$
v^{\prime}=\frac{f(S 2+S 1 \delta z-S 3 \delta x+d y)}{f-(S 3+S 2 \delta x-S 1 \delta y+d z)}
$$

where

$$
\begin{aligned}
& S 1=r_{11} x_{w}+r_{12} y_{w}+r_{13} z_{w}+t_{x} \\
& S 2=r_{21} x_{w}+r_{22} y_{w}+r_{23} z_{w}+t_{y}
\end{aligned}
$$

and

$$
S 3=r_{31} x_{w}+r_{32} y_{w}+r_{33} z_{w}+t_{z}
$$

The image coordinates $(u, v)$ without the displacement of the head are:

$$
u=\frac{f C 1}{f-C 3}
$$

and

$$
v=\frac{f C 2}{f-C 3}
$$

The horizontal and vertical displacement errors, $\varepsilon_{d u_{i}}$ and $\varepsilon_{d v_{i}}$, of point $i$ are:

$$
\begin{aligned}
S 1_{i}= & \cos \phi \cos \theta x_{i}+(\cos \phi \sin \theta \sin \psi-\sin \phi \cos \psi) y_{i} \\
& +(\cos \phi \sin \theta \cos \psi+\sin \phi \sin \psi) z_{i}+t_{x} \\
S 2_{i}= & \sin \phi \cos \theta x_{i}+(\sin \phi \sin \theta \sin \psi+\cos \phi \cos \psi) y_{i} \\
& +(\sin \phi \sin \theta \cos \psi-\cos \phi \sin \psi) z_{i}+t_{y} \\
S 3_{i}= & -\sin \theta x_{i}+\cos \theta \sin \psi y_{i}+\cos \theta \cos \psi z_{i}+t_{z}
\end{aligned}
$$

where $\quad \psi$ is the angle about the $x$ axis

$\theta$ is the angle about the $y$ axis

$\phi$ is the angle about the $z$ axis

$t_{x}$ is the translation along the $x$ axis

$t_{y}$ is the translation along the $y$ axis

$t_{z}$ is the translation along the $z$ axis

$\left(x_{i}, y_{i}, z_{i}\right)$ are the coordinates of the desired point in the world coordinate system

In Eqs. (8) and (9), the displacement errors are in terms of the focal length of the sensor, the three-dimensional world coordinates, $\left(x_{w}, y_{w}, z_{w}\right)$, the translation and orientation parameters of the sensor, and the translational and orientational errors of the active head $[d x, d y, d z, \delta x, \delta y, \delta z]$. As a result, for different threedimensional points projected onto an image plane, the horizontal and vertical displacement errors on the image are not the same even if the focal length of the sensor, and the translational and orientational errors of the manipulator are the same. For instance, two points on a three-dimensional object, $\left(x_{1}, y_{1}, z_{1}\right)$ and $\left(x_{2}, y_{2}, z_{2}\right)$, may have unequal horizontal displacement errors, $\varepsilon_{d u_{1}}$ and $\varepsilon_{d u_{2}}$, and unequal vertical displacement errors, $\varepsilon_{d v_{1}}$ and $\varepsilon_{d v_{2}}$. This implies that the distribution of the displacement error for each projected point in an image is not unique in spite of being generated by the same distribution of translational and orientational errors of the sensor.

$$
\begin{aligned}
& \varepsilon_{d u_{i}}=u_{i}^{\prime}-u_{i}=\frac{\left[\begin{array}{lllllll}
\lambda_{1, i} & \lambda_{2, i} & \lambda_{3, i} & \lambda_{4, i} & \lambda_{5, i} & \lambda_{6, i} & \lambda_{7, i}
\end{array}\right]\left[\begin{array}{lllllll}
\delta x & \delta y & \delta z & d x & d y & d z & 1
\end{array}\right]^{T}}{\left[\begin{array}{llllllll}
\lambda_{15, i} & \lambda_{16, i} & \lambda_{17, i} & \lambda_{18, i} & \lambda_{19, i} & \lambda_{20, i} & \lambda_{21, i}
\end{array}\right]\left[\begin{array}{lllllll}
\delta x & \delta y & \delta z & d x & d y & d z & 1
\end{array}\right]^{T}}
\end{aligned}
$$

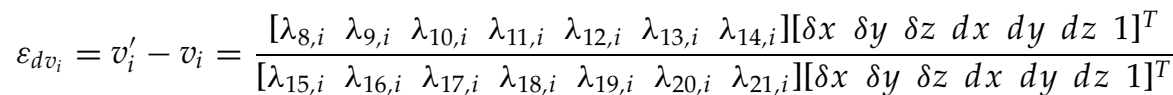

where

$$
\begin{array}{lll}
\lambda_{1, i}=f S 1_{i} S 2_{i} & \lambda_{2, i}=f\left(\left(f-S 3_{i}\right) S 3_{i}-S 1_{i}^{2}\right) & \lambda_{3, i}=f S 2_{i}\left(S 3_{i}-f\right) \\
\lambda_{4, i}=f\left(f-S 3_{i}\right) & \lambda_{5, i}=0 & \lambda_{6, i}=f S 1_{i} \\
\lambda_{7, i}=0 & \lambda_{8, i}=f\left(S 2_{i}^{2}-\left(f-S 3_{i}\right) S 3_{i}\right) & \lambda_{9, i}=-f S 1_{i} S 2_{i} \\
\lambda_{10, i}=f S 1_{i}\left(f-S 3_{i}\right) & \lambda_{11, i}=0 & \lambda_{12, i}=f\left(f-S 3_{i}\right) \\
\lambda_{13, i}=f S 2_{i} & \lambda_{14, i}=0 & \lambda_{15, i}=-S 2_{i}\left(f-S 3_{i}\right) \\
\lambda_{16, i}=S 1_{i}\left(f-S 3_{i}\right) & \lambda_{17, i}=0 & \lambda_{18, i}=0 \\
\lambda_{19, i}=0 & \lambda_{20, i}=f-S 3_{i} & \lambda_{21, i}=\left(f-S 3_{i}\right)^{2}
\end{array}
$$




\section{CONSTRAINT NETWORKS}

\subsection{Hierarchical Constraint Networks and Constraint Functions}

A constraint network is a double, $\mathrm{CN}(\mathbf{X}, C)$ where $\mathbf{X}$ represents a set of variables, $\left\{\mathbf{X}_{1}, \mathbf{X}_{2}, \ldots, \mathbf{X}_{\mathbf{n}}\right\}$ and $C$ represents a set of constraints, $\left\{C_{1}, C_{2}, \ldots, C_{m}\right\}$. The nodes in the constraint network represent variables or constraints. A constraint has multiple inputs and a single output (MISO) and can be represented as a triple, $C_{i}(U, k, f())$. $U$ is the set of indexes for the input variables and $k$ is the index of the output variable for the constraint $C_{i} . f()$ represents the constraint function for $C_{i}$. For example, if $U=\{1,2\}$ and $k=3$, and the constraint function of $C_{i}$ is multiply(), then $C_{i}$ represents $\mathbf{X}_{3}=\operatorname{multiply}\left(\mathbf{X}_{1}, \mathbf{X}_{2}\right)$. The arcs in the constraint network represent the relationship between the variables and the constraints. The constraint functions correspond to the relationships between the output variable and the input variables in the constraints. The constraint functions are n-ary mathematical operation and are represented as $\mathbf{X}_{\mathbf{k}}=f\left(\mathbf{X}_{\mathbf{1}}, \mathbf{X}_{\mathbf{2}}, \ldots, \mathbf{X}_{\mathbf{n}}\right)$ where $\mathbf{X}_{1}, \mathbf{X}_{2}, \ldots, \mathbf{X}_{\mathbf{n}}$ are the input variables and $\mathbf{X}_{\mathbf{k}}$ is the output variable. In the hierarchical constraint networks, there are multiple levels of variables, and there are constraint functions between each adjacent pair of levels of variables.

In addition to the real valued constraint functions, the interval valued constraint functions are utilized to propagate the interval values from their input variables to other variables in the constraint networks. Because some of the input and output variables have interval values, and general real valued constraint functions cannot propagate the intervals of the input variables to the intervals of the output variables, interval constraint functions are necessary. Based on the constraints between the variables and the properties of interval arithmetic, the interval constraint functions between the corresponding intervals, $X_{k}=F\left(X_{1}, X_{2}, \ldots, X_{n}\right)$, can be derived.

An interval constraint function relates the input variables and the output variable by means of interval arithmetic, which is an extension of real arithmetic. To deal with variable $\mathbf{X}$, interval constraint functions use closed intervals, $X=\left[x_{\text {low }}, x_{\text {up }}\right]$, which represent $\left\{x \mid x_{\text {low }} \leq x \leq x_{\text {up }}\right\}$. An interval constraint function for the generic arithmetic operation, $\oplus$, is defined as follows ${ }^{3,6}$ :

$$
\begin{aligned}
X \oplus Y & =\left[x_{\text {low }}, x_{\text {up }}\right] \oplus\left[y_{\text {low }}, y_{\text {up }}\right] \\
& =\left\{x \oplus y \mid x_{\text {low }} \leq x \leq x_{\text {up }}, y_{\text {low }} \leq y \leq y_{\text {up }}\right\}
\end{aligned}
$$

The problem we are interested in is as follows:

Given a set of constraints that relate a set of variables having interval or real values in the constraint network, how can the real valued variables be adjusted in order to satisfy the constraint network while all the interval valued variables are unchanged?

\subsection{Satisfaction of Constraint Network}

The definition of satisfaction in the constraint network always depends on the purpose of the application. A good understanding of the goal of constraint satisfaction for the problem being investigated and an appropriate definition of satisfaction for the constraint network are fundamental to a successful application of constraint networks to solve a problem.

In ICSPs, ${ }^{3,6}$ satisfaction of the network is defined in terms of the consistency of the variables, and the purpose of the ICSP is expressed to be refinement of the intervals of the variables as far as possible without losing possible exact solutions of the constraints. However, this definition does not suit the purpose of our application, which is to adjust all the input and output variables of the constraints (with real values) such that the interval propagated from the input variables based on the constraint functions is a subset of the output variable interval. The definition of constraint network satisfaction in our problem should therefore be modified and it should be described in terms of the consistency of the constraints.

\subsubsection{Satisfaction of Interval Constraint Networks Based on Variable Consistency}

In [6], the satisfaction of the interval constraint network is defined based on satisfaction of variables, as follows:

A variable, $\mathbf{X}_{\mathbf{i}}$, is consistent if and only if $\forall\left(x_{i} \in X_{i} \mid \mathbf{X}_{\mathbf{i}}=x_{i}\right), \exists\left(x_{1} \in X_{1}, \ldots, x_{i-1} \in\right.$ $X_{i-1}, x_{i+1} \in X_{i+1}, \ldots, x_{n} \in X_{n} \mid \mathbf{X}_{1}=x_{1}, \ldots$, $\left.\mathbf{X}_{\mathbf{i}-\mathbf{1}}=x_{i-1}, \mathbf{X}_{\mathbf{i}+\mathbf{1}}=x_{i+1}, \ldots, \mathbf{X}_{\mathbf{n}}=x_{n}\right)$, such that all constraints are satisfied.

The constraint network is satisfied if and only if all variables are consistent.

In other words, given a constraint network with $n$ variables, $\mathbf{X}_{\mathbf{1}}$ to $\mathbf{X}_{n}$, and the constraint between a variable, $\mathbf{X}_{\mathbf{i}}$, and the other variables described by 
$\mathbf{X}_{\mathbf{i}}=f\left(\mathbf{X}_{\mathbf{1}}, \ldots, \mathbf{X}_{\mathbf{n}}\right), \mathbf{X}_{\mathbf{i}}$ is consistent if and only if $X_{i} \subseteq$ $F\left(X_{1}, \ldots, X_{n}\right)$. The purpose of the ICSP is to refine the intervals of the variables in the constraint network as far as possible without losing possible exact solutions of the constraints. For the application in this article, it is desirable to have the interval computed using the input intervals and the interval constraint function be a subset of the assigned output interval for each constraint in the network.

The properties of the consistency of a variable as described in ${ }^{6}$ are not appropriate for the problems studied here. This can be illustrated by considering the following constraint function.

$$
\varepsilon=a \varepsilon_{A}+b \varepsilon_{B}
$$

where $\varepsilon$ is the tolerance of an error propagated from the tolerances from the errors $\varepsilon_{A}$ and $\varepsilon_{B}$ $a$ and $b$ are constant

Given the tolerance intervals $\varepsilon, \varepsilon_{A}$, and $\varepsilon_{B}$, the objective is to ensure that the error propagated from $\varepsilon_{A}$ and $\varepsilon_{B}$ does not fall out of $\varepsilon$; that is, $a \varepsilon_{A}+b \varepsilon_{B}$ must be a subset of $\varepsilon$. However, even given that all the variables which have interval values are consistent according to the definition of ICSP $\left(\varepsilon \subseteq a \varepsilon_{A}+b \varepsilon_{B}, \varepsilon_{A} \subseteq\right.$ $\left(\varepsilon-b \varepsilon_{B}\right) / a$, and $\left.\varepsilon_{B} \subseteq\left(\varepsilon-a \varepsilon_{A}\right) / B\right)$, the condition that $a \varepsilon_{A}+b \varepsilon_{B} \subseteq \varepsilon$ is not guaranteed to be satisfied. For instance, given $\varepsilon_{A}=[1.95,2.05], \varepsilon_{B}=[0.98,1.02]$, $\varepsilon=[9.78,10.22], a=4$, and $b=2, \varepsilon, \varepsilon_{A}$ and $\varepsilon_{B}$ are all consistent.

$$
\begin{aligned}
{[9.78,10.22] } & \subseteq 4[1.95,2.05]+2[0.98,1.02] \\
& =[9.76,10.24] \varepsilon \text { is consistent } \\
{[1.95,2.05] } & \subseteq([9.78,10.22]-2[0.98,1.02]) / 4 \\
& =[1.935,2.065] \varepsilon_{A} \text { is consistent } \\
{[0.98,1.02] } & \subseteq([9.78,10.22]-4[1.95,2.05]) / 2 \\
& =[0.79,1.21] \varepsilon_{B} \text { is consistent }
\end{aligned}
$$

However, because $4[1.95,2.05]+2[0.98,1.02]=[9.76$, $10.24] \not \subset[9.78,10.22]$, the tolerance of $\varepsilon$ is not satisfied. Although all the variables which have interval values are consistent, the real-world tolerance requirement is not satisfied with respect to the application in this problem.

\subsubsection{Satisfaction of Constraint Networks with Real and Interval Values Based on Constraint Consistency}

Since the purpose of our application in a constraint network is different from the purpose of ICSP, a new definition of satisfaction is required. The satisfaction of the network depends on the consistency of the components in the network. While in ICSP, the satisfaction of the network is defined in terms of the consistency of the variables. Here, constraint consistency should be used.

The definitions of consistency of a constraint and satisfaction of the constraint network with real and interval values are as follows:

Definition 1: A constraint, $C_{i}(U, k, f())$, is consistent if and only if

$$
\bigcap_{j \in U}\left(\forall x_{j} \in X_{j} \mid \mathbf{X}_{\mathbf{j}}=x_{j}\right), \quad\left(\exists x_{k} \in X_{k} \mid \mathbf{X}_{\mathbf{k}}=x_{k}\right)
$$

such that $C_{i}$ is satisfied, (i.e., $x_{k}=f\left(\ldots, x_{j}, \ldots\right)$ where $j \in U$ ), and where $U$ is the set of indexes for the input variables and $k$ is the index of the output variable for the constraint $C_{i}$.

Definition 2: The constraint network with real and interval values is satisfied if and only if all the constraints are consistent.

For satisfaction of a constraint network, the interval computed using the input values and an interval constraint function should be a subset of the assigned output interval for each constraint in the network. Taking the earlier example with $\varepsilon=[9.78,10.22], \varepsilon_{A}=$ $[1.95,2.05]$, and $\varepsilon_{B}=[0.98,1.02]$, if $a=2$, and $b=6$, then all tolerances are satisfied.

\section{PROPAGATION IN A CONSTRAINT NETWORK WITH REAL AND INTERVAL VALUES}

For this new approach to constraint network satisfaction given in Definition 1, new propagation techniques must be developed. With such techniques, real and interval values can be propagated from variable to variable in the constraint network to ensure that all of the constraints are consistent. To make the constraints consistent, propagation is utilized to update the values of the variables in the network. Values can be propagated from the input values of a constraint to the single output interval, which is known as forward propagation. Alternatively, values can be propagated from the single output interval to multiple inputs, which is known as backward propagation. Given a set of inputs, forward propagation techniques are used to check whether the propagated output defines a consistent constraint. If the constraint is not consistent, the backward propagation techniques are used to adjust the real valued inputs to achieve consistency. 


\subsection{Forward Propagation}

Forward propagation is based on the constraint function such that the interval or real values of the input variables are propagated to the interval value of the single output variable. If the interval propagated from the input values is not a subset of the output interval, the constraint is not consistent. The algorithm for forward propagation is: constraint function with respect to the corresponding variable and the width of the output interval. The absolute change in the real value is directly proportional to the width of the output interval, but inversely proportional to the derivative of the constraint function with respect to the corresponding variable. Because it is possible for the constraint function to have more than one real-valued input variable, each

Forward Propagation for constraint, $C(\{1,2, \ldots, n\}, k, f()), \operatorname{FP}\left(\mathbf{X}_{\mathbf{1}}, \mathbf{X}_{\mathbf{2}}, \ldots, \mathbf{X}_{\mathbf{n}} ; \mathbf{X}_{\mathbf{k}}\right)$

Propagation from Input Variable to the Upper Limit of the Output Interval $x_{k_{\text {up }}}^{\prime}=f\left(x_{1 \varphi}, \ldots, x_{n \varphi}\right)$ where $x_{i \varphi}=x_{i_{\text {up }}}$

$x_{i \varphi}=x_{i_{\text {low }}}$ if $d f / d \mathbf{X}_{\mathbf{i}}>0$; that is, $\mathbf{X}_{\mathbf{k}}$ is increasing with respect to $\mathbf{X}_{\mathbf{i}}$ $x_{i \varphi}=x_{i}$ if $d f / d \mathbf{X}_{\mathbf{i}}<0$; that is, $\mathbf{X}_{\mathbf{k}}$ is decreasing with respect to $\mathbf{X}_{\mathbf{i}}$ if $\mathbf{X}_{\mathbf{i}}$ has real value

Propagation from Input Variable to the Lower Limit of the Output Interval

$$
\begin{aligned}
x_{k_{\text {low }}}^{\prime}=f\left(x_{1 \kappa}, \ldots, x_{n \kappa}\right) & \\
\text { where } x_{i \kappa}=x_{i_{\text {low }}} & \text { if } d f / d \mathbf{X}_{\mathbf{i}}>0 \\
x_{i \kappa}=x_{i_{\text {up }}} & \text { if } d f / d \mathbf{X}_{\mathbf{i}}<0 \\
x_{i \kappa}=x_{i} & \text { if } \mathbf{X}_{\mathbf{i}} \text { has real value }
\end{aligned}
$$

\section{Checking the Consistency of the Constraint}

$$
\begin{aligned}
& \text { If } x_{k_{\text {up }}^{\prime}}^{\prime}>x_{k_{\text {up }}} \text { or } x_{k_{\text {low }}}^{\prime}<x_{k_{\text {low }}} \\
& \text { then } C(\{1,2, \ldots, n\}, k, f()) \text { is not consistent. }
\end{aligned}
$$

\subsection{Backward Propagation}

Backward propagation is also based on the constraint function such that the interval of the output variable is propagated to the real values of the input variables. When a forward propagation identifies a constraint that is not consistent, the output interval is propagated back to the input intervals by adjusting the real values of the inputs. These values will increase or decrease depending on the partial derivative of the variable value is increased or decreased by a limited amount at every iteration until the constraint is satisfied. The magnitude of the changes is controlled by a weight. Before adjusting the real-valued variables, a pair of constraint inequalities are first constructed for the upper limit and lower limit of the output interval based on the constraint function under consideration. The algorithm for backward propagation through a single constraint is given as follows:

Backward Propagation for constraint, $C(\{1,2, \ldots, n\}, k, f()), \mathrm{BP}\left(\mathbf{X}_{\mathbf{k}} ; \mathbf{X}_{\mathbf{1}}, \mathbf{X}_{\mathbf{2}}, \ldots, \mathbf{X}_{\mathbf{n}}\right)$

Set up a pair of constraint inequalities for $C(\{1,2, \ldots, n\}, k, f())$

Ineq1: $f\left(x_{1 \varphi}, \ldots, x_{n \varphi}\right) \leq x_{k_{\mathrm{up}}}$

where $x_{i \varphi}=x_{i_{\text {up }}} \quad$ if $d f / d \mathbf{X}_{\mathbf{i}}>0$

$x_{i \varphi}=x_{i_{\text {low }}} \quad$ if $d f / d \mathbf{X}_{\mathbf{i}}<0$

$x_{i \varphi}=\mathbf{X}_{\mathbf{i}} \quad$ if $\mathbf{X}_{\mathbf{i}}$ has real value

Ineq2: $f\left(x_{1 \kappa}, \ldots, x_{n \kappa}\right) \geq x_{k_{\text {low }}}$

where $x_{i \kappa}=x_{i_{\text {low }}} \quad$ if $d f / d \mathbf{X}_{\mathbf{i}}>0$

$x_{i \kappa}=x_{i_{\text {up }}} \quad$ if $d f / d \mathbf{X}_{\mathbf{i}}<0$

$x_{i \kappa}=\mathbf{X}_{\mathbf{i}} \quad$ if $\mathbf{X}_{\mathbf{i}}$ has real value 


\section{Adjusting the Real Values of the Input Variables}

For $i=1$ to $n$ (where $n$ is the number of input variables of the constraint)

(1) If Ineq1 is not satisfied Increase $x_{i \varphi}$ by $\Delta x_{i \varphi} \quad$ if $\mathbf{X}_{\mathbf{i}}$ has real value and $d f / d \mathbf{X}_{\mathbf{i}}<0$ Decrease $x_{i \varphi}$ by $\Delta x_{i \varphi} \quad$ if $\mathbf{X}_{\mathbf{i}}$ has real value and $d f / d \mathbf{X}_{\mathbf{i}}>0$

(2) If Ineq2 is not satisfied Increase $x_{i \kappa}$ by $\Delta x_{i \kappa} \quad$ if $\mathbf{X}_{\mathbf{i}}$ has real value and $d f / d \mathbf{X}_{\mathbf{i}}>0$ Decrease $x_{i \kappa}$ by $\Delta x_{i \kappa} \quad$ if $\mathbf{X}_{\mathbf{i}}$ has real value and $d f / d \mathbf{X}_{\mathbf{i}}<0$

( $\Delta x_{i \varphi}$ and $\Delta x_{i \kappa}$ depend on the gradient of $f\left(x_{1 \varphi}, \ldots, x_{n \varphi}\right)$ and $f\left(x_{1 \kappa}, \ldots, x_{n \kappa}\right)$ with respect to $x_{i}$.) Repeat until Ineq1 and Ineq2 are both satisfied.

$\Delta x_{i \varphi}$ and $\Delta x_{i \kappa}$ are computed as follows:

$\Delta x_{i \varphi}=\frac{w_{i}\left(X_{k_{\text {up }}}-X_{k_{\text {low }}}\right)}{\frac{d f\left(X_{1 \varphi}, \ldots, X_{\text {nq }}\right)}{d \mathbf{X}_{\mathbf{i}}}}$ and $\Delta x_{i \kappa}=\frac{w_{i}\left(X_{k_{\text {up }}}-X_{k_{\text {low }}}\right)}{\frac{d f\left(X_{1 \kappa}, \ldots, X_{n k}\right)}{d \mathbf{X}_{\mathbf{i}}}}$

where $w_{i}$ is the weight, $\mathbf{X}_{\mathbf{k}}$ is the output variable, and $f\left(\mathbf{X}_{1}, \ldots, \mathbf{X}_{\mathbf{n}}\right)$ is the constraint function.

Backward propagation from one level to a lower level through a set of constraints is similar to the backward propagation through a single constraint, except that propagation through all constraints between two adjacent levels in the network is processed simultaneously instead of by satisfying each constraint separately. Between two adjacent levels of the network, the variables in the lower level may be input variables for one or more constraints for which the output variable is one of the variables at the next level. Separate backward propagation for each constraint may not produce a solution that satisfies the consistency requirement for all involved constraints simultaneously. For example, assume $\mathbf{X} 3$ is an input variable for constraints $C 1$ and $C 2$ for which $\mathbf{X} \mathbf{1}$ and $\mathbf{X} \mathbf{2}$ are the output variables, respectively. If backward propagations are processed from $\mathbf{X} \mathbf{1}$ through $\mathrm{C} 1$ and from $\mathbf{X} \mathbf{2}$ through $\mathrm{C} 2$ to $\mathrm{X} 3$ separately, the first propagation may produce the value $x 3_{1}$ and the second propagation may produce the value $x 3_{2}$ for $X 3$. Choosing either of these two solutions can easily cause the other constraint to be inconsistent. A technique for backward propagating all the higher level variables to all the lower level variables values through all the constraints simultaneously can be described as follows:

Backward Propagation between Two Adjacent Levels with constraints

$C 1(\{1,2, \ldots, n\}, k)$ to $C m(\{1,2, \ldots, n\}, k)$

Set up a set of constraint inequalities for $C 1(\{1,2, \ldots, n\}, k)$ to $C m(\{1,2, \ldots, n\}, k)$

Set up Ineq $1_{j}$ and Ineq $2_{j}$ using the first step of backward propagation on a single constraint for $j=1,2, \ldots, m$ (where $j$ is the index of the constraints).

\section{Adjusting the Real Values of the Input Variables on the Lower Level}

(1) For $j=1$ to $m$ (where $m$ is the number of constraints and $j$ is the index of the constraints)

For $i=1$ to $n$ (where $n$ is the number of input variables)

(1a) If Ineq $1_{j}$ is not satisfied

Increase $x_{i \varphi}$ by $\Delta x_{i \varphi, j} \quad$ if $\mathbf{X}_{\mathbf{i}}$ has real value and $d f_{j} / d \mathbf{X}_{\mathbf{i}}<0$

Decrease $x_{i \varphi}$ by $\Delta x_{i \varphi, j} \quad$ if $\mathbf{X}_{\mathbf{i}}$ has real value and $d f_{j} / d \mathbf{X}_{\mathbf{i}}>0$

(1b) If Ineq $2_{j}$ is not satisfied

Increase $x_{i \kappa}$ by $\Delta x_{i \kappa, j} \quad$ if $\mathbf{X}_{\mathbf{i}}$ has real value and $d f_{j} / d \mathbf{X}_{\mathbf{i}}>0$

Decrease $x_{i \kappa}$ by $\Delta x_{i \kappa, j} \quad$ if $\mathbf{X}_{\mathbf{i}}$ has real value and $d f_{j} / d \mathbf{X}_{\mathbf{i}}<0$

( $\Delta x_{i \varphi, j}$ and $\Delta x_{i \kappa, j}$ depends on the gradient of $f_{j}\left(x_{1 \varphi}, \ldots, x_{n \varphi}\right)$ and $f_{j}\left(x_{1 \kappa}, \ldots, x_{n \kappa}\right)$ with respect to $x_{i}$.)

Repeat (1) until Ineq $1_{j}$ and Ineq $2_{j}$ are all satisfied for $j=1,2, \ldots, m$. 


\section{MOTION PLANNING TO SATISFY THE TOLERANCE CONSTRAINT}

A constraint network can be utilized to determine the motion of the end-effector that satisfies the allowed tolerance in location and orientation of the final position of the end-effector. The 95\% confidence interval for the joint translation errors and orientation errors is determined experimentally and is assigned as the interval value for the corresponding variables in the constraint network. Acceptable tolerance in the position of the end-effector is determined based on the task. The robot arm is assigned an initial motion. This initial motion can be generated by a motion planning algorithm. The constraints represent the relationship between the end-effector's resulting position and orientation, and the initial position, the translational and orientational joint errors, and the initial path. Forward propagation is used to check the consistency of the resulting end-effector configuration with specified endeffector tolerances, and backward propagation is used to adjust the path if the constraint is not consistent.

\subsection{Cylindrical Coordinate Arm}

Substituting $T^{\prime}$ from Eq. (4) into Eq. (3), three constraint functions that relate the tolerance of the endeffector's position, and the motion, initial position, and the joints' translational and orientational errors can be formulated. The constraint functions in the constraint network are shown in Figure 3 and are as follows:

$$
\begin{aligned}
C 1: x^{\prime}= & \cos (\alpha+\Delta \alpha) x-\sin (\alpha+\Delta \alpha) y \\
& +(t r+\Delta t r) \cos (\alpha+\Delta \alpha+\beta) \\
C 2: y^{\prime}= & \sin (\alpha+\Delta \alpha) x+\cos (\alpha+\Delta \alpha) y \\
& +(t r+\Delta t r) \sin (\alpha+\Delta \alpha+\beta) \\
C 3: z^{\prime}= & z+t z+\Delta t z
\end{aligned}
$$

$\Delta \alpha, \Delta t r$, and $\Delta t z$ are possible joint errors and their ranges or statistical characteristics are determined experimentally. For example, the robot arm can be assigned a particular move a hundred times and its errors in rotation about the $z$ axis and translations along the radial direction and along the $z$ axis are recorded. The resulting 95\% confidence interval from this data can then be used for $\Delta \alpha, \Delta t r$, and $\Delta t z$ intervals. These intervals are fixed and represent the error characteristics of the robot arm system.

Let's assume that $\Delta \alpha=[-0.0005,0.0005]$ (unit is $\mathrm{rad}$ ), $\Delta t r=\Delta t z=[-0.05,0.05]$ (unit is $\mathrm{mm}$ ). It is

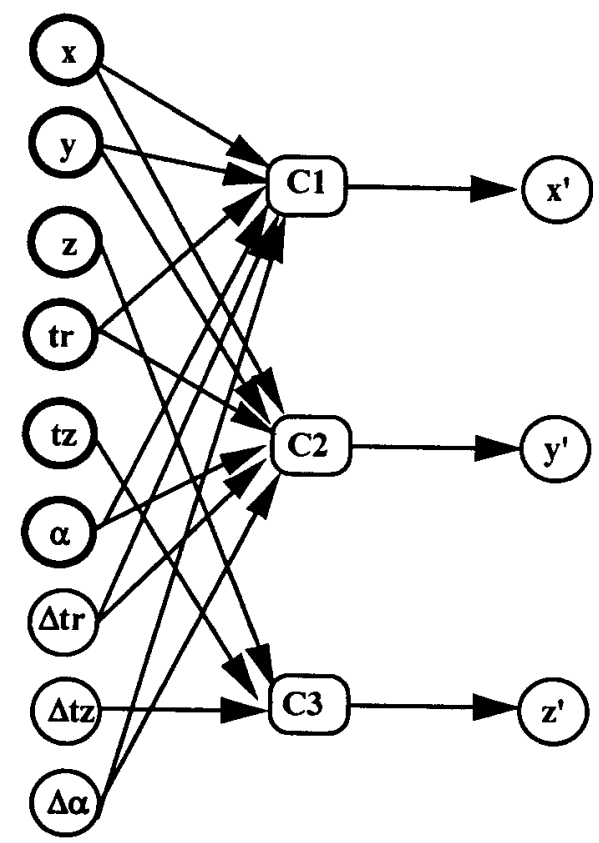

Figure 3. The constraint network for a cylindrical coordinate arm.

given that the initial position of the end-effector, $[x, y, z]=[9.40,3.42,120.00]\left(\right.$ or $[r, \beta, z]=\left[10.00,20^{\circ}\right.$, 120.00]), and the assigned joint motions are $\alpha=60^{\circ}$, $t r=100.00, t z=200.00$. Also assume the desired tolerances for $x^{\prime}, y^{\prime}$, and $z^{\prime}$ are $\left[19.00\left(=x_{\text {low }}^{\prime}\right)\right.$, $\left.19.15\left(=x_{\text {up }}^{\prime}\right)\right],\left[108.17\left(=y_{\text {low }}^{\prime}\right), 108.32\left(=y_{\text {up }}^{\prime}\right)\right], \quad$ and [319.95 $\left.\left(=z_{\text {low }}^{\prime}\right), 320.10\left(=z_{\text {up }}^{\prime}\right)\right]$.

In terms of the constraint network in Figure 3 and the described forward propagation technique, $C 1$ and $C 2$ are found to be inconsistent. (Propagated intervals of $x^{\prime}$ and $y^{\prime}$ are [19.04, 19.16] and [108.27, 108.39]. $19.16>x_{\text {up }}^{\prime}$ and $108.39>y_{\text {up }}^{\prime}$.) Therefore, backward propagations are required to adjust the real values of variables $\alpha$ and $t r$.

Since $x_{\text {low }}^{\prime}<19.04$ and $y_{\text {low }}^{\prime}<108.27$, only two inequalities are needed for backward propagation using $C 1$ and $C 2$ :

$$
\begin{aligned}
& \cos \left(\alpha+\Delta \alpha_{\mathrm{low}}\right) x-\sin \left(\alpha+\Delta \alpha_{\mathrm{low}}\right) y \\
& \quad+\left(t r+\Delta t r_{\mathrm{up}}\right) \cos \left(\alpha+\Delta \alpha_{\mathrm{low}}+\beta\right) \geq x_{\mathrm{up}}^{\prime} \\
& \sin \left(\alpha+\Delta \alpha_{\mathrm{up}}\right) x+\cos \left(\alpha+\Delta \alpha_{\mathrm{up}}\right) y \\
& \quad+\left(t r+\Delta t r_{\mathrm{up}}\right) \sin \left(\alpha+\Delta \alpha_{\mathrm{up}}+\beta\right) \geq y_{\mathrm{up}}^{\prime}
\end{aligned}
$$

Substituting $\Delta \alpha_{\text {low }}=-0.0005 \mathrm{rad}, \Delta \alpha_{\text {up }}=0.0005 \mathrm{rad}$, $\Delta t r_{\text {up }}=0.05, x=9.49, y=3.42, \beta=60^{\circ}, x_{\text {up }}^{\prime}=19.15$, 
$y_{\text {up }}^{\prime}=108.32$, in inequalities (13) and (14):

$$
\begin{gathered}
9.40 \cos (\alpha-0.0005 \mathrm{rad})-3.42 \sin (\alpha-0.0005 \mathrm{rad}) \\
+(t r+0.05) \cos (\alpha-0.0005 \mathrm{rad}+\pi / 9) \leq 19.15
\end{gathered}
$$

$9.40 \sin (\alpha+0.0005 \mathrm{rad})+3.42 \cos (\alpha+0.0005 \mathrm{rad})$

$+(\operatorname{tr}+0.05) \sin (\alpha-0.0005 \mathrm{rad}+\pi / 9) \leq 108.32$

Using the backward propagation between two adjacent levels of constraint network, $\alpha$ increases by $w_{\alpha, x_{\text {up }}}(0.00046)$ and $t r$ decreases by $w_{t r, x_{\text {up }}}(0.985)$ using inequality (13) in each iteration. $\alpha$ increases by $w_{\alpha, y_{\text {up }}}(0.00787)$ and $t r$ increases decreases by $w_{t r, y_{\text {up }}}$ (0.152) using inequality (14) in each iteration. For each iteration, substituting 0.01 to $w_{\alpha, x_{\text {up }}}, w_{t r, x_{\text {up }}}, w_{\alpha, y_{\text {up }}}$, and $w_{t r, y_{\text {up }}}$. After several iterations, $\alpha$ is adjusted to $60.01^{\circ}$ and $t r$ is adjusted to 99.92 so that $\mathrm{C} 1$ and $\mathrm{C} 2$ become consistent $\left(x^{\prime}=[19.01,19.13], y^{\prime}=[108.20,108.31]\right)$.

\subsection{Displacement Errors in Active Vision}

In order to measure an edge segment on a component, the length of the corresponding line segment projected onto the image plane is dimensioned. $\varepsilon_{d u_{i}}$ and $\varepsilon_{d v_{i}}$, the horizontal and vertical displacement errors, depend on the orientation and location of the sensor, the location of the edge segment, and the translational and orientational errors $(\delta x, \delta y, \delta z, d x, d y$, and $d z$ ). Assuming the two endpoints of the desired edge segment are $\left(x_{1}, y_{1}, z_{1}\right)$ and $\left(x_{2}, y_{2}, z_{2}\right)$ in the world coordinate system, the endpoints projected onto the image plane are $\left(u_{1}, v_{1}\right)$ and $\left(u_{2}, v_{2}\right)$ and the horizontal and vertical displacement errors of the line endpoints are $\varepsilon_{d u_{1}}, \varepsilon_{d v_{1}}, \varepsilon_{d u_{2}}$, and $\varepsilon_{d v_{2}}$. A constraint network is constructed as shown in Figure 4. The

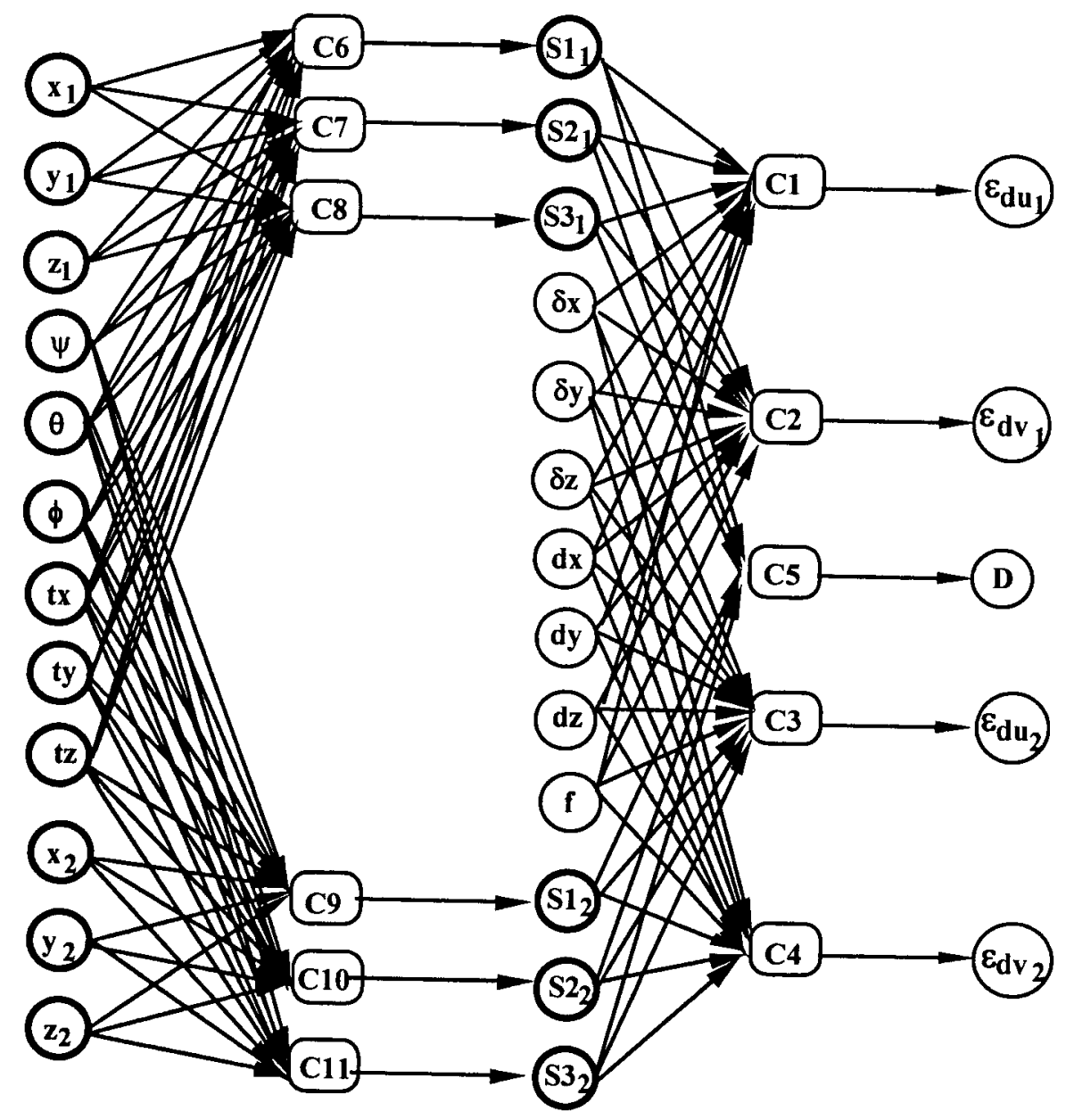

Figure 4. The constraint network for the displacement errors of active vision inspection on a edge segment. 
constraint functions are based on Eqs. (8) through (12). $\mathrm{C} 1$ and $\mathrm{C} 3$ are derived using Eq. (8), C2 and C4 are derived using Eq. (9), C6 and C9 are derived using Eq. (10), C7 and C10 are derived using Eq. (11), and $\mathrm{C} 8$ and $\mathrm{C} 11$ are derived using Eq. (12).

Similar to the experiments with the robot manipulators, $\delta x, \delta y, \delta z, d x, d y$, and $d z$ are determined experimentally. Let's assume $\delta x=\delta y=\delta z=[-0.0005$, 0.0005] (unit is rad) and $d x=d y=d z=[-0.05,0.05]$ (unit is mm). Given the end points of the desired edge segments in the world coordinates are $\left[x_{1}, y_{1}, z_{1}\right]=$ $[10.00,0.00,0.00]$ and $\left[x_{2}, y_{2}, z_{2}\right]=[-10.00,0.00$, $0.00]$, the orientations of the camera are $\psi=-40^{\circ}$, $\theta=-45^{\circ}, \phi=-20^{\circ}$, and the translations of the camera are $t x=56.85, t y=19.04, t z=-597.00$, the tolerances for the displacement errors in the projected end points on the image are $\varepsilon_{d u_{1}}=\varepsilon_{d v_{1}}=\varepsilon_{d u_{2}}=\varepsilon_{d v_{2}}=$ $[-0.0150,0.0150]$.

Using the constraint network in Figure 4 and the described forward propagation technique, $\mathrm{C} 2$ and $\mathrm{C} 4$ are found to be inconsistent $\left(\varepsilon_{d v_{1}}=[-0.0153,0.0153]\right.$ and $\left.\varepsilon_{d v_{2}}=[-0.0151,0.0151]\right) . \mathrm{C} 1$ and C3 are found to be consistent because $\varepsilon_{d u_{1}}=[-0.0147,0.0147]$ and $\varepsilon_{d u_{2}}=[-0.0147,0.0147]$. Using the backward propagation technique, the camera is relocated such that $t x$ is adjusted to $41.74, t y$ is adjusted to 17.22 , and $t z$ is adjusted to -609.21 . C1, C2, C3, and C4 are now all consistent where $\varepsilon_{d u_{1}}=[-0.0145,0.0145], \varepsilon_{d v_{1}}=$ $[-0.0150,0.0150], \varepsilon_{d u_{2}}=[-0.0146,0.0146]$, and $\varepsilon_{d v_{2}}=$ $[-0.0148,0.0148]$. The displacement errors resulting from the new camera setting have a smaller interval than those from the initial camera setting, therefore, a higher accuracy in dimensional measurement can be obtained.

\section{CONCLUSION}

Constraint networks can often be an important tool in engineering applications. In order to effectively exploit constraints and constraint networks in different engineering applications, a good understanding of the purpose of constraints and what they represent in the investigated problem is essential. There are different types of constraint networks that are appropriate for different engineering applications. Therefore, appropriate definitions for constraint consistency and network satisfaction must be developed to fit each engineering application. Techniques for forward and backward propagation should be developed subsequently based on the constraint consistency and the network satisfaction definitions. In this work, constraint networks with real and interval values are described with their potential applications in robotics and active visual inspection. The contributions of this work can be summarized as follows:

- Appropriate definitions for consistency of constraints and satisfaction of such real and interval-valued constraint networks are developed for some classes of applications in mechanical design and robotics.

- Forward and backward propagation techniques for the described constraint networks having real- and interval-valued variables are presented. The presented methods adjust the realvalued variables in the constraint network in order to make all involved constraints consistent without changing the interval-valued variables.

- Applications illustrating use of the techniques in (i) robot manipulators, and (ii) displacement errors in active visual inspection are presented.

\section{REFERENCES}

1. J. Chen and L.M. Chao, Positioning error analysis for robot manipulators with all rotary joints, IEEE Int Conf on Robotics and Automation, San Francisco, CA, April, 1986.

2. F.W. Ciarallo, C.C. Yang, and M.M. Marefat, Displacement errors in active visual inspection, Proc IEEE Int Conf on Robotics and Automation, Minneapolis, MN, April, 1996.

3. E. Davis, Constraint propagation with interval labels, Artif Intell 32 (1987), 281-331.

4. R. Dechter and J. Pearl, Network-based heuristics for constraint-satisfaction problems, Artif Intell 34 (1988), $1-3$.

5. R. Dechter and J. Pearl, Tree clustering for constraint networks, Artif Intell 38 (1989), 353-366.

6. E. Hyvonen, Constraint reasoning based on interval arithmetic: The tolerance propagation approach, Artif Intell 58:(1-3) (1992), 71-112.

7. P.B. Ladkin and A. Reinefeld, Effective solution of qualitative interval constraint problems, Artif Intell 57 (1992), 105-124.

8. A.K. Mackworth and E.C. Freuder, The complexity of some polynomial network consistency algorithms for constraint satisfaction problems, Artif Intell 25 (1985), 65-74.

9. J.-M. Renders, E. Rossignol, M. Becquet, and R. Hanus, Kinematic calibration and geometrical parameter identification for robots, IEEE Trans Robot Automat 7:(6) (1991).

10. R.C. Smith and P. Chessman, On the representation and estimation of spatial uncertainty, Int J Robot Res 5:(4) (1986).

11. S.-F. Su and C.S.G. Lee, Manipulation and propagation of uncertainty and verification of application of actions 
in assembly tasks, IEEE Trans Syst Man Cybernet 22:(6) (1992).

12. C.C. Yang and F.W. Ciarallo, Optimized sensor placement for active visual inspection, J Robot Syst 18:(1) (2001), 1-15.

13. C.C. Yang, M.M. Marefat, and F.W. Ciarallo, Interval constraint networks for tolerance analysis and synthesis, Artif Intell Eng Des Anal Manufact (Special Issue on AI in Manufacturing: State of the Art) 14:(4) (2000), 271-287.
14. C.C. Yang, M.M. Marefat, and F.W. Ciarallo, Analysis of errors and planning accuracy on dimensional measurement in active vision inspection, IEEE Trans Robot Automat 14:(3) (1998), 476-487.

15. C.C. Yang, M.M. Marefat, and F.W. Ciarallo, Analysis of errors in dimensional inspection based on active vision, Proc SPIE on Intelligent Robots and Computer Vision XIII: 3D Vision, Product Inspection and Active Vision, Boston, MA, October 31-November 4, 1994. 